\title{
Magnetic contributions in Bekenstein type models
}

\author{
Lucila Kraiselburd, ${ }^{1,2, *}$ Florencia L. Castillo, ${ }^{3, \dagger}$ Mercedes E. Mosquera, ${ }^{2,4,5, \$}$ and Héctor Vucetich ${ }^{1, \S}$ \\ ${ }^{1}$ Grupo de Astrofísica, Relatividad y Cosmología, Facultad de Ciencias Astronómicas y Geofísicas, \\ Universidad Nacional de La Plata, Paseo del Bosque S/N, 1900 La Plata, Argentina \\ ${ }^{2}$ CONICET, Godoy Cruz 2290, 1425 Ciudad Autónoma de Buenos Aires, Argentina \\ ${ }^{3}$ Instituto de Física Corpuscular, Consejo Superior de Investigaciones Científicas y Universitat de \\ València, Parque Científico, C/Catedrático José Beltrán 2, 46980 Paterna, España \\ ${ }^{4}$ Facultad de Ciencias Astronómicas y Geofísicas, Universidad Nacional de La Plata, \\ Paseo del Bosque S/N, 1900 La Plata, Argentina \\ ${ }^{5}$ Departamento de Física, Universidad Nacional de La Plata, C.C. 67, 1900 La Plata, Argentina
}

(Received 22 August 2017; published 22 February 2018)

\begin{abstract}
In this work, we analyze the spatial and time variation of the fine structure constant $(\alpha)$ upon the theoretical framework developed by Bekenstein (Phys. Rev. D 66, 123514 (2002)). We have computed the field $\psi$ related to $\alpha$ at first order of the weak-field approximation and have also improved the estimation of the nuclear magnetic energy and, therefore, their contributions to the source term in the equation of motion of $\psi$. We obtained that the results are similar to the ones published in L. Kraiselburd and H. Vucetich, Int. J. Mod. Phys. E 20, 101 (2011) which were computed using the zero order of the approximation, showing that one can neglect the first order contribution to the variation of the fine structure constant. Through the comparison between our theoretical results and the observational data of the Eötvös-type experiments or the time variation of $\alpha$ over the cosmological time scale, we set constraints on the free parameter of the Bekenstein model, namely the Bekenstein length.
\end{abstract}

DOI: 10.1103/PhysRevD.97.043526

\section{INTRODUCTION}

Both theories that have been developed to try to unify the fundamental interactions (see, for example, [1-6]) as Dirac's large numbers hypothesis (LNH) [7] predict that the fundamental constants might vary in spacetime. Given these theoretical motivations, there have been many attempts to look for observational and/or experimental data to corroborate such variations (see Refs. [8-11] and references therein) but almost all of them are until now consistent with a null variation. On the other hand, the analysis of the data performed in Refs. [12-16] suggests that the fundamental constants might have a different value than the present ones. A space variation of fundamental constants would produce a violation of the weak equivalence principle (WEP) [17] which can be verified thanks to the energy conservation [18]. The Eötvös-type experiments are the most sensitive methods to verify the WEP since they are capable to detect the

\footnotetext{
*1krai@fcaglp.unlp.edu.ar

†casflo@ific.uv.es

${ }^{*}$ mmosquera@fcaglp.unlp.edu.ar

${ }^{\S}$ vucetich@fcaglp.unlp.edu.ar
}

Published by the American Physical Society under the terms of the Creative Commons Attribution 4.0 International license. Further distribution of this work must maintain attribution to the author(s) and the published article's title, journal citation, and DOI. Funded by SCOAP. difference between the acceleration of bodies with different composition or structure [19-24]. For details on the variation of the fundamental constants see [25].

Bekenstein proposed a theory to study the fine structure constant variability based on first principles [26] and later, he claimed that no violations of WEP are perceived whereas a possible time variation of $\alpha$ can not be discarded with the existed observable bounds [27]. Kraiselburd and Vucetich [28] modified Bekenstein's original theory by replacing the classical model of charged particles by a quantum model of many bodies at the nonrelativistic limit, but with classical fields and neglecting the contributions of the self-generated fields. They found that even considering the cancellation proposed by Bekenstein, between the electric field and the mass source term, quantum density fluctuations produce fluctuating electric currents which generate magnetic fields. These fields, are responsible for the appearance of an anomalous term in the acceleration of a system of particles that produces a violation of the WEP in Eötvös-type experiments. Later, Barrow and Magueijo [29,30] questioned Kraiselburd and Vucetich's interpretation of approaching the coupling of field $\psi$ in the expressions $\boldsymbol{D}=\epsilon \boldsymbol{E}=e^{-2 \psi} \boldsymbol{E}$ and $\boldsymbol{H}=\mu^{-1} \boldsymbol{B}=$ $e^{-2 \psi} \boldsymbol{B}$ with the weak-field approximation $\left(e^{-2 \psi} \approx 1\right)$.

The purpose of this work is twofold:

(1) to show that nonlinear corrections to the zero order in Bekenstein's equations are negligible in the weakfield regime, typical for experimental setups; 
(2) to analyze the prediction for the time variation of $\alpha$ due to the magnetic contributions in the semiclassical model and compare it with modern observational data.

This work is organized as follows. In Sec. II, we briefly review the formalism of Bekenstein model and Kraiselburd and Vucetich approach. In Sec. III, we introduce the improvements to the semiclassical model in order to study the spatial dependence, as well as the time variation of the fine structure constant in an expanding Universe. The observational available data on the variation of the $\alpha$ are shown in Sec. IV. In Sec. V, we present the obtained bounds on the model parameters through the comparison between the observational data and the theoretical predictions. Finally, in Sec. VI, we draw the conclusions.

\section{A REVIEW ON BEKENSTEIN'S FORMALISM AND THE SEMICLASSICAL APPROXIMATION}

In this section, we summarize the main aspects of the Bekenstein model $[26,27]$ and its semiclassical version presented in [28].

\section{A. Bekenstein model}

Bekenstein modifies Maxwell's electromagnetic theory to introduce a scalar field $\psi$ that couples to charges generating a variation in $\alpha[26,27]$. The model holds under the following hypothesis

(1) Maxwell's theory must be recovered when $\alpha$ is constant;

(2) the dynamic field $\psi$ is responsible for changes in $\alpha$;

(3) the dynamics of the electromagnetic and the $\psi$ fields can be obtained from a variational principle;

(4) the theory must be gauge and time-reversal invariant, and also preserve causality;

(5) it is not possible to have a length smaller than the Planck length $\ell_{P}{ }^{1}$

From these hypotheses, the only possible action is given by

$$
S=S_{\mathrm{em}}+S_{\psi}+S_{\mathrm{mat}}+S_{G},
$$

where the expressions for the modified Maxwell and $\psi$ field actions are

$$
\begin{gathered}
S_{\mathrm{em}}=-\frac{1}{16 \pi} \int e^{-2 \psi} f^{\mu \nu} f_{\mu \nu} \sqrt{-g} d^{4} x, \\
S_{\psi}=\frac{-\hbar c}{2 \ell_{B}^{2}} \int\left(\partial_{\mu} \psi\right)^{2} \sqrt{-g} d^{4} x,
\end{gathered}
$$

\footnotetext{
${ }^{1}$ In string theories or in a low-energy limit of some TOEs (theory of everything), this statement should not be taken into account since, for these kind of theories, there are length scales that are smaller than the Planck scale. In these cases, this hypothesis should be revised.
}

$$
\begin{gathered}
S_{\mathrm{mat}}=\sum_{i} \int \frac{1}{\gamma}\left[-m c^{2}+e \frac{u^{\mu}}{c} A_{\mu}\right] \delta^{3}\left[x^{i}-x^{i}(\tau)\right] d^{4} x, \\
S_{G}=\frac{c^{4}}{16 \pi G_{N}} \int \sqrt{(-g)} R d^{4} x,
\end{gathered}
$$

where $F_{\mu \nu}$ is the usual electromagnetic tensor, $f_{\mu \nu}=$ $e^{\psi} F_{\mu \nu} . g$ is the metric while $\hbar$ and $c$ are the Planck constant and the speed of light respectively. $\ell_{B}$ is the Bekenstein's fundamental length scale, a constant length introduced by the model. The Lorentz factor is represented by $\gamma, m$ is the particle mass, $e$ and $u^{\mu}$ are its electric charge and 4th velocity respectively. Finally, $G_{N}$ is the gravitational constant, $R$ the Ricci scalar, and $x^{i}(\tau)$ stands for the particle trajectory of the particle in presence of the fields $A_{\mu}$ and $\psi$.

Following the previous statements, an elementary electric charge and the fine structure constant $\alpha$ are locally defined as

$$
e\left(x^{\mu}\right)=e_{0} e^{\psi\left(x^{\mu}\right)}, \quad \alpha\left(x^{\mu}\right)=e^{2 \psi\left(x^{\mu}\right)} \alpha_{0},
$$

where $e_{0}, \alpha_{0}$ are the current values of the electron charge and the fine structure constant.

The equations that describe the dynamics of the electromagnetic and $\psi$ fields are

$$
\begin{gathered}
\left(e^{-\psi} F^{\mu \nu}\right)_{, \nu}=4 \pi j^{\mu}, \\
\square \psi=\frac{\ell_{B}^{2}}{\hbar c}\left(\frac{\partial \sigma}{\partial \psi}-\frac{F^{\mu \nu} F_{\mu \nu}}{8 \pi}\right) .
\end{gathered}
$$

In the previous equation $j^{\mu}=e_{0} u^{\mu} \delta^{3}\left[x^{i}-x^{i}(\tau)\right] \times$ $(c \gamma \sqrt{-g})^{-1 / 2}, \sigma$ is the energy density of matter [26], and $\square$ stands for the covariant flat d'Alambertian.

Bekenstein [26] has analyzed the spatial $\left(\nabla^{2} \psi\right)$ and the temporal $\left(\frac{1}{c^{2}} \frac{\partial^{2} \psi}{\partial^{2} t}\right)$ terms of Eq. (4b). The cosmological equation of motion for $\psi$ is related to the time variation of $\alpha$. In an isotropic and homogeneous Universe, with a Robertson-Walker metric, the time variation of $\alpha$ (after calling $\epsilon=e^{\psi}$ and $\left.2|\dot{\epsilon} / \epsilon|=\left|\dot{\alpha} / \alpha_{0}\right|\right)$ is given by

$$
\frac{\partial}{\partial t}\left(a^{3} \frac{1}{\epsilon} \frac{\partial \epsilon}{d t}\right)=\frac{-a^{3} \ell_{B}^{2}}{\hbar c}\left[\epsilon \frac{\partial \sigma}{\partial \epsilon}+\frac{\left(E^{2}-B^{2}\right)}{4 \pi}\right],
$$

where $a$ represents the Universe scale factor. As one can see, the only source term is the one that comes from matter, since the electromagnetic radiation of the Universe does not contribute. Considering that magnetic term can be neglected such that the contribution of the Coulombic energy density is much higher in ordinary matter and $\epsilon\left(\frac{\partial \sigma}{\partial \epsilon}\right) \sim 2 m_{\mathrm{EM}} c^{2}$ 


$$
\frac{\partial}{\partial t}\left(a^{3} \frac{1}{\epsilon} \frac{\partial \epsilon}{d t}\right)=\frac{-a^{3} \ell_{B}^{2}}{c \hbar} \zeta_{C} \rho_{m} c^{2}
$$

where $\rho_{m}$ is the total rest-mass density of electromagnetic interacting matter $\left(m_{\mathrm{EM}}\right)$ and $\zeta_{C}$ is the fractional contribution of the Coulomb energy to the rest mass. Mosquera et al. [31] have analyzed the solution of the above equation for two different regimes of the Universe: when matter and radiation dominate, and when matter and cosmological constant do, matching both solutions with the appropriate boundary conditions. They have performed a statistical analysis to test the solutions using observational data from the early Universe and other bounds from the late Universe arriving at the conclusion that Bekenstein's hypothesis $\ell_{B}>\ell_{P}$ should be relaxed.

In Ref. [26], Bekenstein states that the mass dependent term of Eq. (4b) is almost cancelled with the term of the electric field, therefore $\frac{\partial \sigma}{\partial \psi}+\frac{e^{-2 \psi} E^{2}}{4 \pi} \approx 0$. He justifies this statement with the fact that field $\psi$ can be written as a function of the coulombic potential $\phi_{C}$, and working on this relationship, he derives that there exists an asymptotically a null value of $\psi[27]^{2}$ Hence, an electrically charged particle (or a set of point charges) satisfies $\square \psi \approx 0$, meanwhile for a static system of magnetic dipoles, $\nabla^{2} \psi \approx \frac{-\ell_{B}^{2}}{\hbar c} e^{-2 \psi} \frac{B^{2}}{4 \pi}$ and $\frac{\partial}{\partial t}\left(a^{3} \frac{1}{\epsilon} \frac{\partial \epsilon}{\partial t}\right)=\frac{a^{3} \ell_{B}^{2}}{c \hbar}\left|\zeta_{m}\right| \rho_{m} c^{2}$. The factor $\zeta_{m}$ is the fractional contribution of the magnetic energy to the rest mass. For this latter system, Bekenstein claims that no WEP violation can be detected in laboratory experiments.

\section{B. The semiclassical approach}

In Bekenstein's theory, particles are treated classically. This assumption is not accurate, especially in those cases where quantum effects become relevant, such as white dwarfs or condensed matter physics, or at high-energy scales (a small distance scales) since fermions have their own "natural length scale". For these reasons, Kraiselburd and Vucetich [28] have proposed a semiclassical model where particles are quantized and fields remain classic. Once matter is quantized, the main subject is to understand matter distributions movements inside a scalar field $\psi$, and the contributions of the electric and magnetic field of this interaction.

The full action and Lagrangian of a body (or an ensemble of point charges) have been analyzed in the non-relativistic limit for the charges, with the full expression for the electromagnetic field. If only the external $\psi$ and gravitational fields are taken into account, meaning that the selfgenerated fields are neglected, the equation of motion for the scalar field $\psi$ is

\footnotetext{
${ }^{2}$ If this statement is valid the restrictions found in Ref. [31] cease to make sense since $\frac{\partial}{\partial t}\left(a^{3} \frac{1}{\epsilon} \frac{\partial \epsilon}{\partial t}\right)=0$ considering only the Coulomb contribution.
}

$$
\square \psi=4 \pi \kappa^{2}\left[\frac{\partial(\bar{\Psi} \partial m \Psi)}{\partial \psi}+\frac{F_{\mu \nu} F^{\mu \nu}}{8 \pi}\right],
$$

being $\kappa^{2}=\frac{\ell_{B}^{2}}{4 \pi \hbar c} . \quad \Psi$ represents the charged particle field, while the particle mass has been renormalized as $m=m_{0}+\partial m$ with $m_{0}$ independent of $\psi$. The expression $\partial m$ can only be computed through the quantum electrodynamics renormalization since it arises from the quantum fluctuations of the electromagnetic field, $\partial m \approx \frac{3}{4 \pi} e^{2 \psi} \alpha_{0} m_{0} \log \left(\frac{\Lambda^{2}}{m_{0}^{2}}\right)$, with $\Lambda$ the ultraviolet cut-off frequency imposed by quantum electrodynamics. Under this scheme, it is not possible to determine if Bekenstein's cancellation statement (in this case, $\frac{\partial(\bar{\Psi} \partial m \Psi)}{\partial \psi}+e^{-2 \psi} \frac{E^{2}}{4 \pi} \approx 0$ ) is valid or not since it is necessary to quantify the fields [32].

Even taking Bekenstein's statement as valid, in a quantum model of matter, quantum fluctuations of protons and neutrons oscillating in anti-phase create a dipole moment which produces a variable current and magnetic field. Haugan and Will $[33,34]$ have made estimations of these contributions to the magnetic energy from a minimal nuclear shell model roughly obtaining

$$
E_{m} \simeq \frac{3}{20 \pi} \frac{\hat{E}}{R(A) \hbar c} \int \sigma d E=\frac{0.24}{\pi} \frac{\hat{E}}{R(A) \hbar c},
$$

where $R(A)=1.2 A^{1 / 3}$ fm and $\hat{E} \sim 25 \mathrm{MeV}$ are the nuclear radius and the giant dipole mean absorption energy respectively, and $A$ is the atomic number. The integrated strength function $\int \sigma d E$ satisfies the Thomas-Reiche-Kuhn sum rule but for light nuclei, this approximation is no longer suitable. Consequently, in this article we use other estimates for nitrogen, hydrogen and beryllium (see the Appendix).

The magnetic energy density $e_{m}$, which is concentrated near atomic nucleus, can be written in terms of the fractional contribution of the magnetic energy to the rest mass of nuclear specie $b\left(M_{b}\right), \zeta_{m}^{b}=\frac{E_{m}^{b}}{M_{b} c^{2}}$ as,

$$
\begin{aligned}
e_{m}(\boldsymbol{x}) & =\sum_{a} E_{m}^{a} \delta\left(\boldsymbol{x}-\boldsymbol{x}_{a}\right) \simeq \sum_{b} E_{m}^{b} n_{b}(\boldsymbol{x}) \\
& \simeq \frac{\sum_{b} \zeta_{m}^{b} \rho_{b}(\boldsymbol{x})}{\rho(\boldsymbol{x})} \rho(\boldsymbol{x}) c^{2}=\bar{\zeta}_{m}(\boldsymbol{x}) \rho(\boldsymbol{x}) c^{2}
\end{aligned}
$$

where $\bar{\zeta}_{m}(\boldsymbol{x})=\frac{\sum_{b} \zeta_{m}^{b} \rho_{b}(\boldsymbol{x})}{\rho(\boldsymbol{x})}$ is the local mass-weighted average of $\zeta_{m}$ and $n_{b}(\boldsymbol{x})$ is the particle density of the nuclear specie $b$. Finally, $\rho_{b}(\boldsymbol{x})$ stands for the mass density of the nuclear specie $b$, and $\rho(\boldsymbol{x})$ is the local mass density.

Reference [28] is based specifically on the analysis of the magnetic contributions in the spatial part of a semiclassical Bekenstein model. Taking the spatial part of Eq. (7) and rewriting it using expression of Eq. (9) it becomes 


$$
\nabla^{2} \psi=-8 \pi \kappa^{2} c^{2} e^{-2 \psi} \bar{\zeta}_{m} \rho
$$

A solution for an arbitrary distribution of sources with spherical symmetry of the scalar field $\psi$ under the weakfield approximation $\left(e^{-2 \psi} \sim 1\right)$ can be found as,

$$
\psi(r)=8 \pi \kappa^{2} c^{2} \int_{0}^{R_{s}} \frac{x^{2}}{r_{>}} \bar{\zeta}_{m}(x) \rho(x) d x,
$$

being $R_{s}$ the distribution radius and

$$
r_{>}= \begin{cases}r & \text { if } r>x \\ x & \text { if } x>r\end{cases}
$$

Then, using the Newtonian potential, $\phi_{N}(r)=G_{N} M / r$, the field outside the distribution $(r>x)$ can be written as

$$
\begin{aligned}
\psi(r) & \asymp \frac{8 \pi c^{2} \kappa^{2}}{G_{N} M} \phi_{N}(r) \int_{0}^{R_{s}} x^{2} \bar{\zeta}_{m}(x) \rho(x) d x \\
& =2\left(\frac{\ell_{B}}{\ell_{P}}\right)^{2} \tilde{\zeta}_{m} \frac{\phi_{N}(r)}{c^{2}} .
\end{aligned}
$$

In the last expression, $\tilde{\zeta}_{m}$ is the mass-averaged value of $\bar{\zeta}_{m}$. The Lagrangian of the theory, according to Ref. [28], is

$$
L=-M_{\mathrm{tot}}\left[c^{2}-\frac{V_{C M}^{2}}{2}-\phi_{N}\left(\boldsymbol{R}_{C M}\right)\right]+2 \psi\left(\boldsymbol{R}_{C M}\right) E_{m}+\cdots,
$$

where $M_{\text {tot }}$ is the body mass, $V_{C M}$ is the velocity of the center of mass and $\boldsymbol{R}_{C M}$ is the center of mass position. Recalling the assumption of the cancellation between the electrostatic contribution and mass dependence on $\psi$, one can write the acceleration that suffers a body immersed in an external gravitational $(\boldsymbol{g})$ and $\psi$ fields as

$$
\ddot{\boldsymbol{R}}_{C M}=\boldsymbol{a}=\boldsymbol{g}+\left.2 \frac{E_{m}}{M_{\mathrm{tot}}} \nabla \psi\right|_{C M},
$$

with $\nabla \phi_{N}(r)=g$. The neglected terms in the last expression are of either tidal order, negligible in laboratory tests of WEP, or of higher order in $\psi$. The last term on the righthand side of the above equation is an anomalous acceleration generated by the scalar field $\psi$. Consequently, the validity of the theory can then be tested with Eötvös-type experiments that measure the correlation between inertial mass and gravitational mass. From expressions of Eqs. (12) and (14) it is possible to compute the Eötvös parameter which associated with the differential acceleration of two bodies $(A$ and $B)$ of different composition

$$
\eta(A, B)=\frac{a_{A}-a_{B}}{g}=4\left(\frac{\ell_{B}}{\ell_{P}}\right)^{2} \zeta_{m}^{S}\left(\zeta_{m}^{A}-\zeta_{m}^{B}\right),
$$

TABLE I. Eötvös-type experiments data.

\begin{tabular}{lcccc}
\hline \hline Body $A$ & Body $B$ & Source & $10^{11} \eta(A, B)$ & References \\
\hline $\mathrm{Al}$ & $\mathrm{Au}$ & Sun & $1.0 \pm 1.5$ & {$[19]$} \\
$\mathrm{Al}$ & $\mathrm{Pt}$ & Sun & $0.03 \pm 0.045$ & {$[20]$} \\
$\mathrm{Cu}$ & $\mathrm{W}$ & Sun & $0.0 \pm 2.0$ & {$[21]$} \\
$\mathrm{Be}$ & $\mathrm{Al}$ & Earth & $-0.02 \pm 0.23$ & {$[22]$} \\
$\mathrm{Be}$ & $\mathrm{Cu}$ & Earth & $-0.19 \pm 0.25$ & {$[22]$} \\
$\mathrm{Be}$ & $\mathrm{Al}$ & Sun & $0.40 \pm 0.98$ & {$[22]$} \\
$\mathrm{Be}$ & $\mathrm{Cu}$ & Sun & $-0.51 \pm 0.61$ & {$[22]$} \\
$\mathrm{Si} / \mathrm{Al}$ & $\mathrm{Cu}$ & Sun & $0.51 \pm 0.67$ & {$[22]$} \\
$\mathrm{EC}$ & $\mathrm{MM}$ & Sun & $0.001 \pm 0.032$ & {$[23]$} \\
$\mathrm{Be}$ & $\mathrm{Ti}$ & Earth & $0.004 \pm 0.018$ & {$[24]$} \\
\hline \hline
\end{tabular}

where $\zeta_{m}^{S}, \zeta_{m}^{A}$ and $\zeta_{m}^{B}$ are the magnetic energy fractions of the source and bodies $A$ and $B$ respectively.

The bounds on $\left(\frac{\ell_{B}}{\ell_{P}}\right)^{2}$ obtained in Ref. [28] using the data of the most accurate versions of the Eötvös experiment presented in Table I, are

$$
\left(\frac{\ell_{B}}{\ell_{P}}\right)^{2}=0.0003 \pm 0.0006, \quad \frac{\ell_{B}}{\ell_{P}}<0.05,
$$

where the last equation correspond to the upper bound at $3 \sigma$ level.

From these results it is clear that even when the electric field does not contribute to the scalar field $\psi$, the quantum fluctuations contribute (through currents) in such a way that the upper limit found for the $\ell_{B} / \ell_{P}$ parameter discards the theory.

\section{HIGHER ORDERS IN THE WEAK-FIELD APPROXIMATION UNDER THE SEMICLASSICAL APPROACH}

In this section, we present the computation of the field $\psi$ by taking into account the following order in the series expansion in the equation of motion under the scheme proposed by Kraiselburd and Vucetich [28].

In order to carry out this calculation, we proceed to solve Eq. (10) with an iterative method inspired in the Picard method of solution for differential equations. Considering a weak field, $e^{-2 \psi} \approx 1-2 \psi+O\left(\psi^{2}\right)$, we begin by introducing this approximation of the exponential in the equation of motion [see Eq. (10)]. Then, we replace the scalar field $\psi$ on its right-hand side (source term) with the solution given by Eq. (12), that is the zero order approximation $\psi_{0}(r)$, to find the next order solution $\psi_{1}(r)$,

$$
\begin{aligned}
\nabla^{2} \psi_{1} & =-8 \pi \kappa^{2} c^{2} \bar{\zeta}_{m} \rho\left(1-2 \psi_{0}\right), \\
& =-8 \pi \kappa^{2} c^{2} \bar{\zeta}_{m} \rho\left[1-4\left(\frac{\ell_{B}}{\ell_{P}}\right)^{2} \tilde{\zeta}_{m} \frac{\phi_{N}(r)}{c^{2}}\right],
\end{aligned}
$$

The solution is then 


$$
\begin{aligned}
\psi_{1}(r)= & 8 \pi \kappa^{2} c^{2} \frac{1}{r} \int_{0}^{R} x^{2} \bar{\zeta}_{m}(x) \\
& \times\left[1-4\left(\frac{\ell_{B}}{\ell_{P}}\right)^{2} \tilde{\zeta}_{m} \frac{\phi_{N}(x)}{c^{2}}\right] \rho(x) d x, \\
= & \psi_{0}(r)-\frac{32 \pi \kappa^{2}}{G_{N} M} \phi_{N}(r)\left(\frac{\ell_{B}}{\ell_{P}}\right)^{2} \tilde{\zeta}_{m} \\
& \times \int_{0}^{R} x^{2} \bar{\zeta}_{m}(x) \phi_{N}(x) \rho(x) d x,
\end{aligned}
$$

where $R$ is the radius and $M$ is the mass, both of the source body. The previous integral can be computed as

$$
\int_{0}^{R} x^{2} \bar{\zeta}_{m}(x) \phi_{N}(x) \rho(x) d x=\frac{3}{2} \phi_{N}(R) \tilde{\zeta}_{m} M
$$

where, once again, $\tilde{\zeta}_{m}$ stands for the mass average of $\bar{\zeta}_{m}$. Therefore, the asymptotic behaviour of the field turns out to be

$$
\psi_{1}(r)=\left[1-6\left(\frac{\ell_{B}}{\ell_{P}}\right)^{2} \tilde{\zeta}_{m} \frac{\phi_{N}(R)}{c^{2}}\right] \psi_{o}(r)
$$

For Eötvös-type experiments in which generally the source is the Earth or Sun, the factor $6 \tilde{\zeta}_{m} \frac{\phi_{N}(R)}{c^{2}}$ of the second term inside the bracket is of the order $\sim 2.37 \times$ $10^{-10}$ and $\sim 5.27 \times 10^{-14}$ respectively. Therefore, the contribution of this term would be negligible.

\section{A. Magnetic contributions to the acceleration}

In this section, we compute and discuss the acceleration changes due to the addition of the new computed field $\psi_{1}$. According to Eq. (20)

$$
\begin{aligned}
\left.\nabla \psi_{1}\right|_{\mathrm{CM}}= & 2\left(\frac{\ell_{B}}{\ell_{P}}\right)^{2} \zeta_{m} \frac{\nabla \phi_{N}(r)}{c^{2}} \\
& -12\left(\frac{\ell_{B}}{\ell_{P}}\right)^{4} \zeta_{m}^{2} \frac{\phi_{N}(R) \nabla \phi_{N}(r)}{c^{4}} .
\end{aligned}
$$

Consequently, the acceleration that a body $j$ in presence of a with the Newtonian gravitational and a scalar fields becomes [see Eq. (14)]

$\boldsymbol{a}_{j}=\boldsymbol{g}+4 \zeta_{m}^{j} \zeta_{m}^{S}\left(\frac{\ell_{B}}{\ell_{P}}\right)^{2} \boldsymbol{g}-24 \zeta_{m}^{j} \zeta_{m}^{S} 2\left(\frac{\ell_{B}}{\ell_{P}}\right)^{4} \frac{\phi_{N}(R)}{c^{2}} \boldsymbol{g}$.

One can observe again an anomaly, which is composed of two terms. The first of them agrees with the one described in the previous section [see Eq. (15)], while the second one is the result of the next order of the field $\psi$ expansion described in this work.

\section{B. Magnetic contributions to the time variation of $\alpha$ in an expanding Universe}

In this section, we analyze the time variation of the fine structure constant using the development carried out in Ref. [31] for an expanding Universe. This calculation is performed by taking into account the magnetic contribution of the baryon matter.

From Eq. (5), considering valid Bekenstein's cancellation in the semiclassical approach, we obtain

$$
\frac{1}{\epsilon} \frac{\partial \epsilon}{\partial t}=\frac{3}{4 \pi}\left(\frac{\ell_{B}}{\ell_{P}}\right)^{2} \zeta_{m} \Omega_{b}\left(\frac{a_{0}}{a(t)}\right)^{3} H_{0}^{2}\left(t-t_{c}\right)
$$

where we have used $\rho_{m}=\frac{\Omega_{b} \rho_{C}}{a^{3}(t)}, \rho_{C}=3 /\left(8 \pi G_{N}\right) H_{0}^{2}$ and $\ell_{P}=\left(G_{N} \hbar / c^{3}\right)^{1 / 2}, H_{0}$ is the Hubble constant, $\Omega_{b}$ is the Universe baryon matter density and $t_{c}$ is an integration constant that can be null. One can assume that the field variation is only generated by the magnetic energy of the cosmological baryon matter. If one consider that the primordial Universe is composed only of Hydrogen and Helium-4, the fractional contribution of the magnetic energy to the rest baryon mass is given by

$$
\zeta_{m}=\frac{0.75 E_{m}^{H}+0.25 E_{m}^{{ }^{4} \mathrm{He}}}{0.75 M_{\mathrm{H}} c^{2}+0.25 M_{{ }^{4} \mathrm{He}} c^{2}} \approx 1.097 \times 10^{-5},
$$

where $E_{m}^{H}$ and $E_{m}^{4}$ are the magnetic energy of $\mathrm{H}$ and ${ }^{4} \mathrm{He}$ respectively and $M_{\mathrm{H}}\left(M_{{ }^{4} \mathrm{He}}\right)$ is the $\mathrm{H}\left({ }^{4} \mathrm{He}\right)$ mass. These magnetic energies in particular are estimated differently from the others (an explanation is given in the Appendix).

The scale factor has different expressions depending on the stage of the Universe evolution. In a flat FriedmannRobertson-Walker metric (FRW) and assuming the scalar field contribution null, the equation that describes the Universe scale factor time evolution can be written as

$$
\left(\frac{1}{a} \frac{\partial a}{\partial t}\right)^{2}=H_{0}^{2}\left\{\Omega_{m}\left(\frac{a_{0}}{a(t)}\right)^{3}+\Omega_{r}\left(\frac{a_{0}}{a(t)}\right)^{4}+\Omega_{\Lambda}\right\},
$$

where the initial conditions are $a(0)=0$ and $a\left(t_{0}\right)=1$, being $t_{0}$ the age of the Universe, $\Omega_{m}$ is the matter contribution to the density, $\Omega_{r}$ is the radiation density and $\Omega_{\Lambda}$ stands for the contribution of the cosmological constant to the density. In Ref. [31], the authors have performed a piece-wise approximate solution by solving the FRW equation for two different cases: (a) radiation and matter (early Universe) that can be applied to nucleosynthesis and recombination of primordial hydrogen; and (b) matter and cosmological constant (middle-age and present Universe) which is used for quasar absorption systems, geophysical data, and atomic clocks. Then, they have matched both solutions in the time that the regime changes $\left(t_{1}\right)$ and considered that the scale factor is a smooth and continuous function of time. They have obtained 
(i) early Universe, dominated by radiation and matter $\left(t<t_{1}\right)$

$$
\begin{aligned}
& a_{R M}(\xi)=\frac{\Omega_{m} \xi^{2}}{4}+\sqrt{\Omega_{r}} \xi, \\
& H_{0} t(\xi)=\frac{\Omega_{m} \xi^{3}}{12}+\frac{\sqrt{\Omega_{r}} \xi^{2}}{2} .
\end{aligned}
$$

(ii) middle-age Universe, dominated by matter and cosmological constant $\left(t>t_{1}\right)$

$$
a_{M C}=\sqrt{3} \frac{\Omega_{m}}{\Omega_{\Lambda}}\left[\sinh \left(\frac{3}{2} \sqrt{\Omega_{\Lambda}} H_{0}\left(t-t_{0}\right)+\operatorname{arcsinh}\left(\sqrt{\frac{\Omega_{\Lambda}}{\Omega_{m}}}\right)\right]^{\frac{2}{3}} .\right.
$$

Thereafter, we obtain the time variation of $\alpha$ (being $\ln \frac{\epsilon(t)}{\epsilon\left(t_{0}\right)} \simeq \frac{1}{2} \frac{\Delta \alpha}{\alpha_{0}}$ ) for each stage of the Universe by replacing the scale factor in Eq. (23);

(1) early Universe dominated by radiation and matter $\left(t<t_{1}\right)$

$$
\begin{gathered}
\frac{\Delta \alpha}{\alpha_{0}}=\frac{-1}{\pi} \zeta_{m} \frac{\Omega_{b}}{\Omega_{m}}\left(\frac{\ell_{B}}{\ell_{P}}\right)^{2}\left[\ln \left(\frac{\lambda\left(\xi_{1}\right)}{\lambda(\xi)}\right)+\frac{2 \sqrt{\Omega_{r}}}{\lambda(\xi)}-\frac{2 \sqrt{\Omega_{r}}}{\lambda\left(\xi_{1}\right)}-\frac{1}{8} \ln \left(\frac{\Omega_{r}}{\Omega_{\Lambda}}\right)\right] \\
+\frac{3}{4 \pi} \zeta_{m}\left(\frac{\ell_{B}}{\ell_{P}}\right)^{2} \frac{\Omega_{b}}{\Omega_{r}} H_{0} t_{c}\left[\frac{1}{\xi}-\frac{1}{\xi_{1}}+\frac{\Omega_{m}}{\lambda(\xi)}-\frac{\Omega_{m}}{\lambda\left(\xi_{1}\right)}+\frac{\Omega_{m}}{2 \sqrt{\Omega_{r}}} \ln \left(\frac{\xi \lambda\left(\xi_{1}\right)}{\xi_{1} \lambda(\xi)}\right)\right] \\
-\frac{1}{2 \pi} \zeta_{m} \frac{\Omega_{b}}{\Omega_{m}}\left(\frac{\ell_{B}}{\ell_{P}}\right)^{2} \sqrt{\Omega_{\Lambda}}\left[\frac{\tau_{1}-H_{0} t_{c}}{\tanh \left(\operatorname{arcsinh}\left(\left(\frac{\Omega_{r} \Omega_{\Lambda}}{\Omega_{m}^{4}}\right)^{\frac{3}{8}}\right)\right)}-\frac{\tau_{0}-H_{0} t_{c}}{\tanh \left(\operatorname{arcsinh}\left(\sqrt{\frac{\Omega_{\Lambda}}{\Omega_{m}}}\right)\right)}\right], \\
\lambda(\xi)=\xi \Omega_{m}+4 \sqrt{\Omega_{r}}, \\
\tau_{i}=H_{0} t_{i} .
\end{gathered}
$$

(2) middle-age Universe dominated by matter and cosmological constant $\left(t>t_{1}\right)$

$$
\begin{aligned}
\frac{\Delta \alpha}{\alpha_{0}}= & \frac{-1}{2 \pi} \zeta_{m} \frac{\Omega_{b}}{\Omega_{m}}\left(\frac{\ell_{B}}{\ell_{P}}\right)^{2} \sqrt{\Omega_{\Lambda}} \\
& \times\left[\frac{\tau-H_{0} t_{c}}{\tanh (\gamma(\tau))}-\frac{\tau_{0}-H_{0} t_{c}}{\tanh \left(\operatorname{arcsinh}\left(\sqrt{\frac{\Omega_{\Lambda}}{\Omega_{m}}}\right)\right)}-\frac{2}{3 \sqrt{\Omega_{\Lambda}}} \ln \left(\sqrt{\frac{\Omega_{m}}{\Omega_{\Lambda}}} \sinh (\gamma(\tau))\right)\right],
\end{aligned}
$$

$$
\gamma(\tau)=\frac{3}{2} \sqrt{\Omega_{\Lambda}}\left(\tau-\tau_{0}\right)+\operatorname{arcsinh}\left(\sqrt{\frac{\Omega_{\Lambda}}{\Omega_{m}}}\right) .
$$

(3) present Universe dominated by matter and cosmological constant $\left(t=t_{0}\right)$

$$
\frac{\dot{\alpha}}{H_{0} \alpha_{0}}=\frac{3}{4 \pi} \zeta_{m}\left(\frac{\ell_{B}}{\ell_{P}}\right)^{2} \Omega_{b}\left(\tau_{0}-\tau_{c}\right) .
$$

\section{OBSERVATIONAL DATA ON THE TIME VARIATION OF THE FINE STRUCTURE CONSTANT}

In this section, we present the observational data on the time variation of the fine structure constant. To study this model of the time variation of $\alpha$, we take into account not only the different stages in the Universe's evolution but also use three different sets of cosmological parameters $[9,35]$. Since the baryon density $\left(\Omega_{b} h^{2}\right)$ can be determined by two independent analysis: (i) by the comparison between theoretical calculation of primordial abundances and the observable data; or (ii) by the study of the CMB data (cosmic microwave background) [36-39], we used the results of the analysis of the CMB data. That is $\Omega_{b} h^{2}=0.02212 \pm 0.00028, \quad \Omega_{b} h^{2}=0.0231 \pm 0.0013$ [35], $\Omega_{b} h^{2}=0.0218 \pm 0.0004$ [9].

\section{A. BBN constraints}

During the first three minutes of the Universe the light elements, such as deuterium, ${ }^{4} \mathrm{He},{ }^{3} \mathrm{He},{ }^{7} \mathrm{Li}$, were produced. In order to include the fine structure constant time variation upon the cosmological time scale, we have modified the 
TABLE II. Time variation of the fine structure constant for the different values of the baryon density and considering all data in the sample or removing the ${ }^{7} \mathrm{Li}$ data in the $\chi^{2}$-test.

\begin{tabular}{lcccc}
\hline \hline Model & Data & $\Omega_{b} h^{2}$ & $\frac{\Delta \alpha}{\alpha} \pm \sigma\left[10^{-2}\right]$ & $\chi_{\nu}^{2} /(N-1)$ \\
\hline Model I & All data & $0.0218 \pm 0.0004$ & $0.24_{-0.28}^{+0.30}$ & 3.55 \\
& Without ${ }^{7}$ Li-data & & $-0.32_{-0.32}^{+0.30}$ & 1.24 \\
\multirow{2}{*}{ Model II } & All data & $0.02212 \pm 0.00028$ & $0.52_{-0.26}^{+0.33}$ & 3.54 \\
& Without ${ }^{7}$ Li-data & & $-0.05_{-0.35}^{+0.26}$ & 1.01 \\
\multirow{2}{*}{ Model III } & All data & $0.0231 \pm 0.0013$ & $1.42_{-0.32}^{+0.22}$ & 4.20 \\
& Without ${ }^{7}$ Li-data & & $0.65_{-0.35}^{+0.26}$ & 1.25 \\
\hline \hline
\end{tabular}

numerical code developed by Kawano et al. [40,41] (for the modifications to the code, see [31,42-45] and references therein). As said before, the baryon density was fixed at three different values.

The possible variation of $\alpha$ has been calculated through the comparison of the observable data and the theoretical results using a $\chi^{2}$-test. For the deuterium, we have considered the observable data reported in Refs. [4654]. For ${ }^{4} \mathrm{He}$, we used the data of Refs. [55-63], and the results of Refs. [64-70] for ${ }^{7} \mathrm{Li}$.

In order to check the consistency of the data, we have followed the analysis of Patrignani et al. [71] and increased the observational errors by a factor $\Theta_{\mathrm{D}}=1.28, \Theta_{{ }^{4} \mathrm{He}}=2.79$ and $\Theta_{r_{\mathrm{Li}}}=2.04$. In Table II, we present the results. The inclusion of the lithium data gives a rather poor fit for all the models, meaning for the three values of $\Omega_{b} h^{2}$. If the middle value of the baryon density is used (model II), the fit is excellent and the variation of $\alpha$ is consistent with a nullvariation at $1 \sigma$, if one excludes the ${ }^{7} \mathrm{Li}$ data in the analysis. The fits obtained with the largest (model III) or the smallest (model I) value for $\Omega_{B} h^{2}$ considered in this work, are good fits, however, $\frac{\Delta \alpha}{\alpha}$ is consistent with zero at $2 \sigma$ if the lithium data are removed in the statistical test.

\section{B. CMB constraints}

The recombination process, when the first hydrogen atoms formed and the Universe became transparent to photons, occurred approximately 300000 years after the Big Bang. The photons released during this process are the Cosmic Microwave Background radiation (CMB). This radiation corresponds to a black body radiation at temperature $T_{0}=2.725^{\circ} \mathrm{K}$ with small anisotropies of the order of $10^{-6}{ }^{0} \mathrm{~K}$.

TABLE III. Bounds on the time variation of $\alpha$ from CMB data.

\begin{tabular}{lccc}
\hline \hline Model & Data source & $\frac{\Delta \alpha}{\alpha_{0}} \pm \sigma$ & References \\
\hline Model I & WMAP7 & $-0.016 \pm 0.005$ & {$[9]$} \\
Model II & Planck & $-0.0036 \pm 0.0037$ & {$[35]$} \\
Model III & WMAP9 & $0.007 \pm 0.020$ & {$[35]$} \\
\hline \hline
\end{tabular}

The CMB formation is totally mediated by electromagnetic processes. Thus, a variation in the coupling constant of this interaction (that is $\alpha$ ) involves modifications in the interactions between photons and electrons as well as in the recombination scenario and consequently in the spectrum of CMB fluctuations [9,35,72]. In Table III, we show the bounds obtained in Ref. [9,35] using WMAP7, WMAP9 and Planck satellites data.

\section{Quasars absorption spectra}

Spectroscopic observations of extragalactic objects such as quasars can be used to study the time variation of the fine structure constant. High redshift quasar spectra present absorption resonant lines of alkaline ions whose splits are proportional to $\alpha^{2}$. Therefore, studying the separation of the doublets, one can obtained bounds for the variation of $\alpha$. This analysis (called AD) has been applied, in the literature, to different doublet absorption line systems at different redshifts $[8,73,74]$. The results are consistent with a null variation of the constant.

TABLE IV. Bounds for the time variation of $\alpha$ from quasar absorption systems.

\begin{tabular}{lccc}
\hline \hline Method/quasar & Redshift & $\frac{\Delta \alpha}{\alpha_{0}} \pm \sigma\left[10^{-5}\right]$ & References \\
\hline SIDAM & 1.15 & $0.04 \pm 0.15$ & {$[79]$} \\
SIDAM & 1.15 & $024 \pm 0.38$ & {$[79]$} \\
OH conjugated lines & 025 & $0.5 \pm 1.26$ & {$[80]$} \\
Molecular and radio lines & 0.24 & $-0.10 \pm 0.22$ & {$[81]$} \\
Molecular and radio lines & 0.69 & $-0.08 \pm 0.27$ & {$[81]$} \\
Molecular and radio lines & 0.765 & $<0.67$ & {$[82]$} \\
OH conjugated & 0.865 & $<0.67$ & {$[82]$} \\
$\quad$ and radio lines & & & \\
3 sources & 1.08 & $0.43 \pm 0.34$ & {$[83]$} \\
HE0515-4414 & 1.15 & $-0.01 \pm 0.18$ & {$[84]$} \\
HE0515-4414 & 1.15 & $0.05 \pm 0.24$ & {$[85]$} \\
HE0001-2340 & 1.58 & $-0.15 \pm 0.26$ & {$[86]$} \\
HE1104-1805A & 1.66 & $-0.47 \pm 0.53$ & {$[83]$} \\
HE2217-2818 & 1.69 & $0.13 \pm 0.26$ & {$[86]$} \\
HS1946+7658 & 1.74 & $-0.79 \pm 0.26$ & {$[83]$} \\
Q1101-264 & 1.84 & $0.57 \pm 0.27$ & {$[83]$} \\
\hline \hline
\end{tabular}


TABLE V. Bounds on the time variation of the fine structure constant of three different absorption systems from Quasar HS1549 + 1919 [87].

\begin{tabular}{lcc}
\hline \hline Telescope & Redshift & $\frac{\Delta \alpha}{\alpha_{0}} \pm \sigma_{\text {stat }} \pm \sigma_{\text {sys }}\left[10^{-6}\right]$ \\
\hline Keck/HIRES & 1.143 & $0.20 \pm 13.63 \pm 3.97$ \\
VLT/UVES & 1.143 & $-8.80 \pm 5.60 \pm 4.36$ \\
Subaru/HDS & 1.143 & $-9.04 \pm 10.41 \pm 4.34$ \\
Keck/HIRES & 1.342 & $-2.77 \pm 13.71 \pm 3.16$ \\
VLT/UVES & 1.342 & $0.02 \pm 7.64 \pm 1.85$ \\
Subaru/HDS & 1.342 & $-1.29 \pm 24.04 \pm 6.04$ \\
Keck/HIRES & 1.802 & $-3.92 \pm 8.61 \pm 4.69$ \\
VLT/UVES & 1.802 & $-0.66 \pm 14.65 \pm 4.54$ \\
Subaru/HDS & 1.802 & $-11.20 \pm 7.83 \pm 2.44$ \\
\hline \hline
\end{tabular}

The many multiplet method (MMM) which compares transitions of different species with widely differing atomic masses together with different transitions of the same species, improves the sensitivity, but its systematic errors are generating great controversies. ${ }^{3}$ In Tables IV, V, and VI, we show results obtained by different authors using different methods and absorption lines of different chemical elements.

\section{Oklo constraint}

Oklo is a natural uranium-fusion nuclear reactor that operated $1.8 \times 10^{9}$ years ago in Gabon, Africa. The operating conditions of the reactor can be recovered from the analysis of nuclear and geochemical data to calculate the thermal neutron capture cross sections of several nuclear species including ${ }^{149} \mathrm{Sm},{ }^{151} \mathrm{Eu}$, and ${ }^{155} \mathrm{Gd}$. From these cross sections, it is possible to estimate the value of the resonance energy of the fundamental level at the time of the reaction. A variation of this energy (from the time of the reaction to the present) would reflect a variation in time of $\alpha[10,88-$ 90]. The time variation of the fine structure constant obtained with this natural reactor is [90]

$$
\frac{\Delta \alpha}{\alpha_{0}}=(45 \pm 15) \times 10^{-9} .
$$

\section{E. Long-lived $\boldsymbol{\beta}$ decayers constraints}

Thanks to laboratory measurements and/or by comparison with the age of meteorites, it is possible to determine the half-life of long-lived $\beta$ decayers. Sisterna and Vucetich

\footnotetext{
${ }^{3}$ From the combined observations of distant quasars using the KECK/HIRES [12-15] and VLT/UVES [16] telescopes, there seems to exist substantial evidence for a dipole-type spatial variation of $\alpha$. Pinho and Martins [75] have reached the same conclusion from an independent analysis using the observed data set and others independent observational results. However, Whitmore and Murphy [76] have shown that long-range wavelength distortions can be confused and appreciated as if they were the reported dipolar variation of $\alpha$ [77]. Later, Murphy et al. [78] studied certain transitions that do not suffer from long-range distortions obtaining results consistent with the null variation.
}

TABLE VI. Bounds on the time variation of the fine structure constant from different quasars [78].

\begin{tabular}{lccc}
\hline \hline Quasar & Redshift & Telescope & $\frac{\Delta \alpha}{\alpha_{0}} \pm \sigma_{\text {stat }} \pm \sigma_{\text {sys }}\left[10^{-6}\right]$ \\
\hline J0058 +0041 & 1.072 & Keck & $-1.35 \pm 6.71 \pm 2.51$ \\
J0058 + 0041 & 1.072 & VLT & $17.07 \pm 9.00 \pm 2.41$ \\
PHL957 & 2.309 & Keck & $-0.65 \pm 6.46 \pm 2.26$ \\
PHL957 & 2.309 & VLT & $-0.20 \pm 12.44 \pm 3.51$ \\
J0108 - 0037 & 1.371 & VLT & $-8.45 \pm 5.69 \pm 4.64$ \\
J0226-2857 & 1.023 & VLT & $3.54 \pm 8.54 \pm 2.38$ \\
J0841 + 0312 & 1.342 & Keck & $3.05 \pm 3.30 \pm 2.13$ \\
J0841 + 0312 & 1.342 & VLT & $5.67 \pm 4.19 \pm 2.16$ \\
J1029 + 1039 & 1.622 & Keck & $-1.70 \pm 9.80 \pm 2.47$ \\
J1237 + 0106 & 1.305 & Keck & $-4.54 \pm 8.08 \pm 3.13$ \\
Q1755 + 57 & 1.971 & Keck & $4.72 \pm 4.18 \pm 2.16$ \\
Q2206 - 1958 & 1.921 & VLT & $-4.65 \pm 6.01 \pm 2.24$ \\
\hline \hline
\end{tabular}

[91] have analyzed the dependence between the half-life of these decayers and the fundamental constants $\alpha, \Lambda_{\mathrm{QCD}}$, and $G_{F}$ so, any shift in the half-life may be generated by a variation in these constants at the age of the meteorites and their value now. According to these authors, there is a linear relationship between the shift in the half-life of the $\beta$ decayer of ${ }^{187} \operatorname{Re}$ from the solar system formation up today and the variation of $\alpha$. The transition analyzed was $\mathrm{Re} \rightarrow \mathrm{Os}$ and it is assumed that this event has occurred $4.6 \times 10^{9}$ years ago. According to Ref. [91], the time variation of the fine structure constant is

$$
\frac{\Delta \alpha}{\alpha_{0}}=(-7.4 \pm 7.4) \times 10^{-7} .
$$

\section{F. Atomic clocks constraints}

The time variation of the fine structure constant can be measured by comparing frequencies of atomic clocks with different atomic numbers during time intervals that range from one hundred days to two years. This is possible thanks to the development of very stable frequency oscillators based on hyperfine transitions. These hyperfine levels are determined by the interaction of the nuclear magnetic moment with the magnetic moment of a valence electron.

TABLE VII. Bounds on $\dot{\alpha} / \alpha_{0}$ from different atomic clocks.

\begin{tabular}{lcc}
\hline \hline Frequencies & $\frac{\dot{\alpha}}{\alpha_{0}}\left[10^{-15} \mathrm{yr}^{-1}\right]$ & References \\
\hline $\mathrm{Hg}^{+}$y H maser & $0.0 \pm 14.0$ & {$[92]$} \\
$\mathrm{C}_{\mathrm{s}}$ y $\mathrm{R}_{\mathrm{b}}$ & $8.4 \pm 13.8$ & {$[93]$} \\
$\mathrm{C}_{\mathrm{s}}$ y $\mathrm{R}_{\mathrm{b}}$ & $-0.2 \pm 8.0$ & {$[94]$} \\
$\mathrm{H}_{\mathrm{g}}$ y C & $5.7 \pm 11.2$ & {$[95]$} \\
$\mathrm{Y}_{\mathrm{b}}$ y $\mathrm{C}_{\mathrm{s}}$ & $-1.6 \pm 5.9$ & {$[11]$} \\
$\mathrm{C}_{\mathrm{s}}$ & $0.33 \pm 0.3$ & {$[96]$} \\
$\mathrm{H}_{\mathrm{g}}$ & $0.053 \pm 0.079$ & {$[96]$} \\
$\mathrm{H}_{\mathrm{g}}^{+}$ & $-0.016 \pm 0.023$ & {$[97]$} \\
\hline \hline
\end{tabular}


It is possible to restrict the variation of $\alpha$ by comparing the frequencies of two different atoms because the relativistic contribution to the splitting of the hyperfine levels $\Delta$ grows with the atomic number as $\Delta \propto(Z \alpha)^{2}$. Table VII shows the bounds for the time variation of the fine structure constant presented in the literature.

\section{RESULTS}

In this section, we analyze and discuss the predictions that emerge from the improved Bekenstein model in the semiclassical regime and compare it with experimental and observational data in order to verify the validity of the theory. We analyze separately the spatial and temporal aspects of the theory.

\section{A. WEP experiments for testing the spatial variation of $\alpha$}

As we have explained in Sec. II B, the Eötvös parameter can be obtained from the difference of the acceleration of two different bodies of different composition. In order to determine and/or limit the validity of the theory, we use the data from Eötös-type experiments presented in Table I.

Using Eq. (22), the theoretical prediction of the Eötvös parameter for the semiclassical Bekenstein model is

$$
\begin{aligned}
\eta(A, B)= & \frac{a_{A}-a_{B}}{g}=4\left(\frac{\ell_{B}}{\ell_{P}}\right)^{2} \zeta_{m}^{S}\left(\zeta_{m}^{A}-\zeta_{m}^{B}\right) \\
& -24\left(\frac{\ell_{B}}{\ell_{P}}\right)^{4}\left(\zeta_{m}^{S}\right)^{2}\left(\zeta_{m}^{A}-\zeta_{m}^{B}\right) \frac{\phi_{N}\left(R_{S}\right)}{c^{2}} \\
= & \tilde{A}\left(\frac{\ell_{B}}{\ell_{P}}\right)^{2}+\tilde{B}\left(\frac{\ell_{B}}{\ell_{P}}\right)^{4},
\end{aligned}
$$

where $\tilde{A}$ and $\tilde{B}$ are constant that depend on the composition of the bodies and do not depend on the theoretical parameter of the model. Note that the Eq. (15) can be written as $\eta=\tilde{A}\left(\frac{\ell_{B}}{\ell_{P}}\right)^{2}$. We perform a $\chi^{2}$-test to set constraints on the free parameter of the theory, namely $\ell_{B} / \ell_{P}$, by the comparison of our theoretical results with the observational data. In Table VIII, we show our results and we also compared, in the same table, the results obtained using the approximation of Ref. [28] or the Eq. (15). The differences between this new limit and the one presented in Eq. (16) are due to the improvement in the estimation of the magnetic energy (see the Appendix).

TABLE VIII. Estimated value for the free parameter in two different theoretical expressions. $\chi_{\nu}^{2}$ stand for the normalized $\chi^{2}$.

\begin{tabular}{lcc}
\hline \hline Approximation & $\left(\frac{\ell_{B}}{\ell_{P}}\right)^{2} \pm \sigma$ & $\chi_{\nu}^{2}$ \\
\hline$\eta=\tilde{A}\left(\frac{\ell_{B}}{\ell_{P}}\right)^{2}+\tilde{B}\left(\frac{\ell_{B}}{\ell_{P}}\right)^{4}$ & $(7.9 \pm 70.7) \times 10^{-5}$ & 0.329 \\
$\eta=\tilde{A}\left(\frac{\ell_{B}}{\ell_{P}}\right)^{2}$ & $(7.9 \pm 70.7) \times 10^{-5}$ & 0.329 \\
\hline \hline
\end{tabular}

As one can noticed, the results for both cases are the same, since the contribution from the "next order" (first order) in the series expansion of the field $\psi$ is very small and can be neglected. Consequently, it is valid the use of the zero order approximation with the change on the estimation of the magnetic energy.

Since, at $3 \sigma$ level, the upper bound of the Bekenstein length scale, for both cases, is $\ell_{B}<0.05 \ell_{P}$, it seems natural to discard the theory.

In December 2017, the MICROSCOPE Collaboration published an estimation of $\eta \sim 10^{-15}$ consistent with zero [98]. If this result is used, the $\frac{\ell_{B}}{\ell_{P}}$ bounds would be even smaller.

\section{B. Constraints from the time variation of $\alpha$}

In Sec. III B, we have computed the theoretical predictions for the variation of the fine structure constant in different stages of the Universe. Equations (28a), (29a), and (30) can be written as

$$
\begin{gathered}
\frac{\Delta \alpha}{\alpha}=\breve{A}\left(\frac{\ell_{B}}{\ell_{P}}\right)^{2}+\breve{B} H_{0} t_{c}\left(\frac{\ell_{B}}{\ell_{P}}\right)^{2}, \\
\frac{\dot{\alpha}}{H_{0} \alpha}=\breve{C}\left(\frac{\ell_{B}}{\ell_{P}}\right)^{2}+\breve{C} H_{0} t_{c}\left(\frac{\ell_{B}}{\ell_{P}}\right)^{2},
\end{gathered}
$$

where the constants $\breve{A}, \breve{B}$, and $\breve{C}$ depend only on the cosmological parameters. The constraints on the free parameters of the theory, that is $\left(\frac{\ell_{B}}{\ell_{P}}\right)^{2}$ and $H_{0} t_{c}\left(\frac{\ell_{B}}{\ell_{P}}\right)^{2}$, are obtained by a least squares linear regression using the observational data described in Sec. IV. We have performed a discrimination into different groups of the available data, according to when the physical phenomena took place, that is, (i) early Universe (CMB and BBN bounds), (ii) middleaged Universe (quasars, long-lived $\beta$ decayers, and Oklo reactor bounds); and (iii) present Universe (bounds from atomic clocks). As we have mentioned before, we use three different sets of cosmological parameters.

In Table IX, we present our results for the constraints on the free parameter for the different models (or cosmological parameters) described in Sec. IVA. The value for the variation of the fine structure constant during BBN used in the lineal regression correspond to the obtained in Sec. IVA when the lithium data were discarded in the statistical test. It can be noticed that in the three models, in the cases where the data corresponding to the early Universe are subtracted, the value of $H_{0} t_{c}\left(\frac{\ell_{B}}{\ell_{P}}\right)^{2}$ and its uncertainty increase considerably. In addition, it can be seen that at one standard deviation, Bekenstein length parameter $\ell_{B}$ becomes imaginary for all the cases with the exception of the analysis performed when the middle-aged Universe data are excluded.

An important difference arises from the results for the different groups of cosmological parameters. The estimates 
TABLE IX. Best fits of the free parameters of the model for the different values of the cosmological parameters (see Tables II and III).

\begin{tabular}{lcccc}
\hline \hline Model & Data & $\left(\frac{\ell_{B}}{\ell_{P}}\right)^{2} \pm \sigma$ & $H_{0} t_{c}\left(\frac{\ell_{B}}{\ell_{P}}\right)^{2} \pm \sigma$ & $\chi_{\nu}^{2}$ \\
\hline Model I & All data & $-2.54 \pm 0.84$ & $(-1.82 \pm 1.76) \times 10^{-6}$ & 0.75 \\
& Without early Universe & $-2.54 \pm 0.84$ & $0.053 \pm 1.025$ & 0.56 \\
& Without middle-aged Universe & $-1.05 \pm 2.68$ & $(-1.82 \pm 1.76) \times 10^{-6}$ & 1.29 \\
Model II & Without present Universe & $-2.70 \pm 0.88$ & $(-1.82 \pm 1.76) \times 10^{-6}$ & 0.85 \\
& All data & $-3.55 \pm 1.22$ & $(-2.58 \pm 15.50) \times 10^{-7}$ & 0.57 \\
& Without early Universe & $-3.55 \pm 1.22$ & $0.072 \pm 1.373$ & 0.57 \\
& Without middle-aged Universe & $-1.10 \pm 2.76$ & $(-2.55 \pm 15.50) \times 10^{-7}$ & 0.36 \\
Model III & Without present Universe & $-4.14 \pm 1.35$ & $(-2.57 \pm 15.51) \times 10^{-7}$ & 0.61 \\
& All data & $-4.20 \pm 1.54$ & $(3.66 \pm 1.72) \times 10^{-6}$ & 0.57 \\
& Without early Universe & $-4.20 \pm 1.54$ & $0.084 \pm 1.864$ & 0.60 \\
& Without middle-aged Universe & $-1.11 \pm 2.72$ & $(3.66 \pm 1.72) \times 10^{-6}$ & 0.30 \\
\hline \hline & Without present Universe & $-5.69 \pm 1.87$ & $(3.66 \pm 1.72) \times 10^{-6}$ & 0.60 \\
\hline
\end{tabular}

for $H_{0} t_{c}\left(\frac{\ell_{B}}{\ell_{P}}\right)^{2}$ are negative when we use the results of the cosmological parameters of models I and II, whereas the estimates using the parameters of model III are positive (except the case where BBN and CMB data are excluded).

Since several authors consider the integration constant $t_{c}$ null, we have also performed an estimation of parameter $\left(\frac{\ell_{B}}{\ell_{P}}\right)^{2}$ for each group of cosmological parameters $t_{c}=0$, that is $\frac{\Delta \alpha}{\alpha_{0}}=\breve{A}\left(\frac{\ell_{B}}{\ell_{P}}\right)^{2}$ and $\frac{\dot{\alpha}}{H_{0} \alpha_{0}}=\breve{C}\left(\frac{\ell_{B}}{\ell_{P}}\right)^{2}$. The results presented in Table $\mathrm{X}$ are similar to the previous ones, however the uncertainties are qualitatively greater. This generates that only at $1 \sigma$ level the model does not fulfil the hypothesis imposed on $\ell_{B}$, either because the estimates give imaginary values or smaller values than the Planck length. On the other hand, in the cases where the middle-aged Universe data are eliminated, the model can not be discarded.

TABLE X. Best fits of the free parameters of the model for the different values of the cosmological parameters and $t_{c}=0$ (see Tables II and III).

\begin{tabular}{lccc}
\hline \hline Model & Data & $\left(\frac{\ell_{B}}{\ell_{P}}\right)^{2} \pm \sigma$ & $\chi_{\nu}^{2}$ \\
\hline Model I & All data & $-2.54 \pm 2.22$ & 0.76 \\
& Without early Universe & $-2.54 \pm 2.19$ & 0.55 \\
& Without middle-aged Universe & $-1.05 \pm 4.95$ & 1.27 \\
& Without present Universe & $-2.69 \pm 2.03$ & 0.86 \\
Model II & All data & $-2.99 \pm 2.71$ & 0.56 \\
& Without early Universe & $-2.99 \pm 2.67$ & 0.56 \\
& Without middle-aged Universe & $-1.10 \pm 5.09$ & 0.33 \\
& Without present Universe & $-2.99 \pm 2.19$ & 0.61 \\
Model III & All data & $-2.99 \pm 3.05$ & 0.67 \\
& Without early Universe & $-2.99 \pm 3.01$ & 0.60 \\
& Without middle-aged Universe & $-1.10 \pm 5.02$ & 0.66 \\
& Without present Universe & $-2.99 \pm 2.42$ & 0.74 \\
\hline \hline
\end{tabular}

\section{SUMMARY AND CONCLUSIONS}

In this work, we have analyzed the magnetic contributions in the semiclassical Bekenstein model for the variation of the fine structure constant and show that the magnetic field produces an anomalous term in the acceleration. Based on previous works of Refs. [28,32], we have solved the equation of motion the scalar field $\psi$ to first order (in the mentioned publications this calculation was made at zero order). We have also improved the estimation of nuclear magnetic energy, according to Refs. [99-101]. To set constraints on the Bekenstein's theory parameter, $\ell_{B}$, we have analyzed not only the spatial contribution of the equation of motion of the field related to the fine structure constant, but also its time variation. To perform the last computation we have considered the expansion rate of the Universe in different evolutionary stages.

We have examined possible violations of the weak equivalence principle due to the incorporation of the scalar field. In order to constrain the Bekenstein length, we have compared our theoretical results with the experimental data from Eötvös-type experiments. We conclude that the first term in the series of the weak-field approximation is the one that contributes the most and the following ones can be neglected. Our results are of the same order as those obtained in Ref. [28], and the small differences between them are due only to the change in the estimation of the magnetic energy. Consequently, it can be deduced that the semiclassical model for the spatial variation would be discarded.

We have also analyzed the time variation of the fine structure constant from the semiclassical model of Bekenstein in an expanding Universe. To determine the free parameters of the theory, $\left(\frac{\ell_{B}}{\ell_{P}}\right)^{2}$ and $\left(\frac{\ell_{B}}{\ell_{P}}\right)^{2} H_{0} t_{c}$, we have made a comparison between the observational data for different times with our theoretical predictions. At $3 \sigma$ level, we found 


$$
\begin{aligned}
\left(\frac{\ell_{B}}{\ell_{P}}\right)^{2} & <-0.02, \quad\left(\frac{\ell_{B}}{\ell_{P}}\right)^{2}<0.11, \quad\left(\frac{\ell_{B}}{\ell_{P}}\right)^{2}<0.42, \\
\frac{\ell_{B}}{\ell_{P}} & <0.14 i, \quad \frac{\ell_{B}}{\ell_{P}}<0.33, \quad \frac{\ell_{B}}{\ell_{P}}<0.65,
\end{aligned}
$$

for the different cosmological parameters used, that is, the first constraint corresponding to model I, the second to model II, and the last one to model III. Note that, for the first expression, Bekenstein length turns out to be a nonreal number. These results rule out the theory.

The removal or not of the early Universe data (that is, BBN and $\mathrm{CMB}$ constraints on the variation of the fine structure constant) does not affect the results. The same constraints are obtained if the data removed are those from the present Universe (that is bound obtained from atomic clocks). However, in the case where the data of the middle-aged Universe are not taken into account in the fit, the $3 \sigma$ level upper bound does not allow to discard the theory since $\ell_{B} \sim \ell_{P}$.

The $3 \sigma$ level upper bound results for the parameter $\left(\frac{\ell_{B}}{\ell_{P}}\right)^{2} H_{0} t_{c}$ are (in the same order as before)

$$
\begin{aligned}
& \left(\frac{\ell_{B}}{\ell_{P}}\right)^{2} H_{0} t_{c}<3 \times 10^{-6}, \\
& \left(\frac{\ell_{B}}{\ell_{P}}\right)^{2} H_{0} t_{c}<4 \times 10^{-6}, \\
& \left(\frac{\ell_{B}}{\ell_{P}}\right)^{2} H_{0} t_{c}<8 \times 10^{-6} .
\end{aligned}
$$

We obtained the same results when we excluded data from the middle-aged Universe and/or the present Universe. On the contrary, when the early Universe data are not taken into account the bounds are much less restrictive, that is,

$$
\left(\frac{\ell_{B}}{\ell_{P}}\right)^{2} H_{0} t_{c}<3, \quad\left(\frac{\ell_{B}}{\ell_{P}}\right)^{2} H_{0} t_{c}<4, \quad\left(\frac{\ell_{B}}{\ell_{P}}\right)^{2} H_{0} t_{c}<6 .
$$

Comparing our results with those obtained in Ref. [31] where possible time variations of $\alpha$ due to the Coulomb contributions are analyzed, it is observed that, for the parameter $\left(\frac{\ell_{B}}{\ell_{P}}\right)^{2} H_{0} t_{c}$, both set of bounds are of the same order, while for the parameter $\left(\frac{\ell_{B}}{\ell_{P}}\right)^{2}$, their bounds are more tight (around an order of magnitude or 2 smaller).

Finally, the results for the Bekenstein length when $t_{c}=0$ indicates that only at one standard deviation can the theory be discarded in all cases for the three models except when the data from the middle-aged Universe are not considered in the statistical analysis. Contrasting these results with those obtained from the analyses of the planet's thermal flow (data from the late Universe) [32,102], their $3-\sigma$ upper bounds are of an order of magnitude more strict than ours using data of the whole evolution of the Universe.

It is important to note that almost all the mean values obtained for the parameters $\left(\frac{\ell_{B}}{\ell_{P}}\right)^{2}$ and $\left(\frac{\ell_{B}}{\ell_{P}}\right)^{2} H_{0} t_{c}$ from the analysis of the time variation of $\alpha$ are negative, producing an incongruity in the semiclassical model since the Bekenstein length must be real, positive, and greater than Planck length. This fact gives us a hint of the serious problems that the theory faces despite the given bulging uncertainties.

In conclusion, it has been shown that even taking as valid Bekenstein's statement (about the cancellation in the source term between the contributions of electric field and the mass) in a semiclassical model, the magnetic contributions produce observable anomalous accelerations and time variations of the fine structure constant which are not compatible with the observable data.

\section{ACKNOWLEDGMENTS}

L. K. is supported by PIP 11220120100504 CONICET and, with H. V., by Grant No. G140 UNLP. M. E. M. is supported by Grant No. (PIP-282) of the National Research Council of Argentina (CONICET) and by a research grant of the National Agency for the Promotion of Science and Technology (ANPCYT) of Argentina. L. K. and M. E. M. are members of the Scientific Research Career of the CONICET.

\section{APPENDIX THE INTEGRATED STRENGTH FUNCTION IN THE NUCLEAR MAGNETIC ENERGY}

As mentioned in Sec. II B, magnetic energy density is mainly located close to the atomic nuclei, which is the reason for studying its contributions. Another possible source of magnetic field are the quantum fluctuations of the "number of particles." The main contribution to the magnetic energy under the semiclassical scheme comes from the dipolar nuclear oscillations with $T=1$ (isospin). The protons and neutrons oscillate in antiphase, generating a variable dipole moment and, therefore, a variable current. For a detailed description of the computation of magnetic energy by relating the current to the dipole transition matrix elements with the Thomas-Reiche-Kuhn sum rule, see Ref. [28]. This sum rule is saturated (approximately) by the giant dipole resonance.

In this work, we have a special interest in improving the computation of the integrated strength function, that is, $\int \sigma(E) d E$ of Eq. (8) for different nuclei. For most cases, the rule of Thomas-Reiche-Kuhn [99] gives a good estimate,

$\sigma_{T}=\int \sigma(E) d E=(1+x) \frac{2 \pi^{2} e^{2} \hbar}{m c} \frac{N Z}{A} \simeq 60 \frac{N Z}{A} \mathrm{MeV} \mathrm{mb}$, 
where $x \sim 0.2$ takes into account the interchange and rate of dependence of nuclear interactions, and $N, Z$, and $A$ are the neutron, proton, and mass numbers $(A=N+Z)$ of the element with mass $m$. However, for light nuclei such as hydrogen $(N=0)$, helium $(N=2)$, or beryllium $(N=5$ for $\left.{ }^{9} \mathrm{Be}\right)$, this estimation fails. For this reason, we apply, in these particular cases, the results obtained in Ref. [100], where the effective section of ${ }^{4} \mathrm{He}$ was measured $\left(\sigma_{T} \approx 7.94 \mathrm{MeVmb}\right)$ and, in Ref. [101], where the proton resonances were analyzed with the result $\sigma_{T} \approx 46 \mathrm{MeV} \mathrm{mb}$. On the other hand, from the photodisintegration of ${ }^{9} \mathrm{Be}$ through the $1 / 2+$ state near the neutron threshold analysis [103], the best estimate obtained is $\sigma_{T} \approx 13.3 \mathrm{MeV} \mathrm{mb}$.
[1] Y. Wu and Z. Wang, Phys. Rev. Lett. 57, 1978 (1986).

[2] K. Maeda, Mod. Phys. Lett. A 03, 243 (1988).

[3] T. Damour and A. M. Polyakov, Nucl. Phys. B423, 532 (1994); T. Damour, F. Piazza, and G. Veneziano, Phys. Rev. Lett. 89, 081601 (2002); T. Damour, F. Piazza, and G. Veneziano, Phys. Rev. D 66, 046007 (2002).

[4] G. A. Palma, Ph. Brax, A. C. Davis, and C. van de Bruck, Phys. Rev. D 68, 123519 (2003); Ph. Brax, C. van de Bruck, A. C. Davis, and C. S. Rhodes, Astrophys. Space Sci. 283, 627 (2003).

[5] T. Kaluza, Sitzungsber. Preuss. Akad. Wiss. Berlin (Math. Phys.) 1921, 966 (1921); O. Klein, Z. Phys. 37, 895 (1926).

[6] S. Weinberg, Phys. Lett. B 125, 265 (1983).

[7] P. A. M. Dirac, Nature (London) 139, 323 (1937); P. A. M. Dirac, Proc. R. Soc. A 165, 199 (1938).

[8] H. Chand, P. Petitjean, R. Srianand, and B. Aracil, Astron. Astrophys. 430, 47 (2005).

[9] E. Menegoni, M. Archidiacono, E. Calabrese, S. Galli, C. J. A. P. Martins, and A. Melchiorri, Phys. Rev. D 85, 107301 (2012).

[10] Y. Fujii, A. Iwamoto, T. Fukahori, T. Ohnuki, M. Nakagawa, H. Hidaka, Y. Oura, and P. Möller, Nucl. Phys. B 573, 377 (2000).

[11] E. Peik, B. Lipphardt, H. Schnatz, T. Schneider, Chr. Tamm, and S. G. Karshenboim, Phys. Rev. Lett. 93, 170801 (2004).

[12] J. K. Webb, V. V. Flambaum, C. W. Churchill, M. J. Drinkwater, and J. D. Barrow, Phys. Rev. Lett. 82, 884 (1999).

[13] J. K. Webb, M. T. Murphy, V. V. Flambaum, V. A. Dzuba, J. D. Barrow, C. W. Churchill, J. X. Prochaska, and A. M. Wolfe, Phys. Rev. Lett. 87, 091301 (2001).

[14] M. T. Murphy, J. K. Webb, V. V. Flambaum, J. X. Prochaska, and A. M. Wolfe, Mon. Not. R. Astron. Soc. 327, 1237 (2001).

[15] M. T. Murphy, J. K. Webb, J. K., and V. V. Flambaum, Mon. Not. R. Astron. Soc. 345, 609 (2003).

[16] J. A. King, J. K. Webb, M. T. Murphy, V. V. Flambaum, R. F. Carswell, M. B. Bainbridge, M. R. Wilczynska, and F. Elliott Koch, Mon. Not. R. Astron. Soc. 422, 3370 (2012).

[17] C. W. Misner, K. S. Thorne, and J. A. Wheeler, Gravitation (W. H. Freeman, San Francisco, 1973).

[18] M. P. Haugan, Ann. Phys. (N.Y.) 118, 156 (1979).
[19] P. G. Roll, R. Krotkov, and R. H. Dicke, Ann. Phys. (N.Y.) 26, 442 (1964).

[20] V. B. Braginskii and V. I. Panov, Sov. Phys. JETP 34, 463 (1972).

[21] G. M. Keiser and J.E. Faller, in Proc. 2nd Marcel Grossman Meeting on General Relativity, edited by R. Ruffini (North-Holland Publishing Company, Amsterdam, 1982), p. 969.

[22] Y. Su, B. R. Heckel, E. G. Adelberger, J. H. Gundlach, M. Harris, G. L. Smith, and H. E. Swanson, Phys. Rev. D 50, 3614 (1994).

[23] S. Baeßler, B. R. Heckel, E. G. Adelberger, J. H. Gundlach, U. Schmidt, and H. E. Swanson, Phys. Rev. Lett. 83, 3585 (1999).

[24] S. Schlamminger, K.-Y. Choi, T. A. Wagner, J. H. Gundlach, and E. G. Adelberger, Phys. Rev. Lett. 100, 041101 (2008).

[25] J. P. Uzan, Living Rev. Relativ. 14, 2 (2011).

[26] J. D. Bekenstein, Phys. Rev. D 25, 1527 (1982).

[27] J. D. Bekenstein, Phys. Rev. D 66, 123514 (2002).

[28] L. Kraiselburd and H. Vucetich, Int. J. Mod. Phys. E 20, 101 (2011).

[29] J. Barrow and J. Magueijo, Phys. Rev. D 90, 123506 (2014).

[30] J. Barrow and J. Magueijo, Mod. Phys. Lett. A 30, 1540029 (2015).

[31] M. E. Mosquera, C. G. Scóccola, S. J. Landau, and H. Vucetich, Astron. Astrophys. 478, 675 (2008).

[32] L. Kraiselburd, Ph.D. thesis, Universidad Nacional de La Plata, Argentina, 2012.

[33] M. P. Haugan and C. M. Will, Phys. Rev. D 15, 2711 (1977).

[34] C. M. Will, Theory and Experiment in Gravitational Physics (Cambridge University Press, Cambridge, England, 1981).

[35] P. A. R. Ade et al. (Planck Collaboration), Astron. Astrophys. 580, A22 (2015).

[36] P. A. R. Ade et al. (Planck Collaboration), Astron. Astrophys. 594, A13 (2016).

[37] P. A. R. Ade et al. (Planck Collaboration), Astron. Astrophys. 571, A16 (2014).

[38] G. Hinshaw, D. Larson, E. Komatsu, D. N. Spergel, C. L. Bennett, J. Dunkley, M. R. Nolta, M. Halpern, R. S. Hill, N. Odegard et al., Astrophys. J. Suppl. Ser. 208, 19 (2013).

[39] E. Komatsu, J. Dunkley, M. R. Nolta, C. L. Bennett, B. Gold, G. Hinshaw, N. Jarosik, D. Larson, M. Limon, L. 
Page, D. N. Spergel et al., Astrophys. J. Suppl. Ser. 180, 330 (2009).

[40] L. Kawano, Report No. fERMILAB-PUB-88-034-A 1988.

[41] L. Kawano, Report No. fERMILAB-PUB-92-004-A 1992.

[42] K. M. Nollett and R. E. Lopez, Phys. Rev. D 66, 063507 (2002).

[43] L. Bergström, S. Iguri, and H. Rubinstein, Phys. Rev. D, 60, 045005 (1999).

[44] S. J. Landau, M. E. Mosquera, and H. Vucetich, Astrophys. J. 637, 38 (2006).

[45] S. J. Landau, M. E. Mosquera, C. G. Scóccola, and H. Vucetich, Phys. Rev. D 78, 083527 (2008).

[46] M. Pettini and D. V. Bowen, Astrophys. J. 560, 41 (2001).

[47] J. M. O’Meara, D. Tytler, D. Kirkman, N. Suzuki, J. X. Prochaska, D. Lubin, and A. M. Wolfe, Astrophys. J. 552, 718 (2001).

[48] D. Kirkman, D. Tytler, N. Suzuki, J. M. O’Meara, and D. Lubin, Astrophys. J. Suppl. Ser. 149, 1 (2003).

[49] J. M. O’Meara, S. Burles, J. X. Prochaska, G. E. Prochter, R. A. Bernstein, and K. M. Burgess, Astrophys. J. Lett. 649, L61 (2006).

[50] C. M. Oliveira, H. Warren Moos, P. Chayer, and J. W. Kruk, Astrophys. J. 642, 283 (2006).

[51] N. H. M. Crighton, J. K. Webb, A. Ortiz-Gil, and A. Fernández-Soto, Mon. Not. R. Astron. Soc. 355, 1042 (2004).

[52] R. J. Cooke et al. (to be published).

[53] R. J. Cooke, M. Pettini, R. A. Jorgenson, M. T. Murphy, and C. C. Steidel, Astrophys. J. 781, 31 (2014).

[54] M. Pettini and R. Cooke, Mon. Not. R. Astron. Soc. 425, 2477 (2012).

[55] Y. I. Izotov, T. X. Thuan, and G. Stasinska, Astrophys. J. 662, 15 (2007).

[56] Y. I. Izotov and T.X. Thuan, Astrophys. J. 602, 200 (2004).

[57] Y. I. Izotov and T. X. Thuan, Astrophys. J. 500, 188 (1998).

[58] T.X. Thuan and Y. I. Izotov, Space Sci. Rev. 100, 263 (2002).

[59] S. Villanova, G. Piotto, and R. G. Gratton, Astron. Astrophys. 499, 755 (2009).

[60] Y. I. Izotov, G. Stasínska, and N. G. Guseva, Astron. Astrophys. 558, A57 (2013).

[61] E. Aver, K. A. Olive, R. L. Porter, and E. D. Skillman, J. Cosmol. Astropart. Phys. 11 (2013) 017.

[62] Y. I. Izotov, T. X. Thuan, and N. Guseva, Mon. Not. R. Astron. Soc. 445, 778 (2014).

[63] V. Luridiana, A. Peimbert, M. Peimbert, and M. Cervino, Astrophys. J. 592, 846 (2003).

[64] P. Bonifacio, L. Pasquini, F. Spite, A. Bragaglia, E. Carretta, V. Castellani, M. Centuriòn, A. Chieffi, R. Claudi, G. Clementini et al., Astron. Astrophys. 390, 91 (2002).

[65] A. M. Boesgaard, M. C. Novicki, and A. Stephens, IAU Symp. 1, 29 (2005).

[66] P. Molaro, P. Bonifacio, and L. Pasquini, Mon. Not. R. Astron. Soc. 292, L1 (1997).

[67] J. Meléndez, L. Casagrande, I. Ramírez, M. Asplund, and W. J. Schuster, Astron. Astrophys. 515, L3 (2010).

[68] A. Mucciarelli, M. Salaris, P. Bonifacio, L. Monaco, and S. Villanova, Mon. Not. R. Astron. Soc. 444, 1812 (2014).
[69] P. E. Nissen and W. J. Schuster, Memorie della Societa Astronomica Italiana Supplementi 22, 41 (2012).

[70] K. Lind, F. Primas, C. Charbonnel, F. Grundahl, and M. Asplund, Astron. Astrophys. 503, 545 (2009).

[71] C. Patrignani et al. (Particle Data Group), Chin. Phys. C 40, 100001 (2016).

[72] S. J. Landau, Ph.D. thesis, Universidad Nacional de La Plata, Argentina, 2002.

[73] L. L. Cowie and A. Songaila, Astrophys. J. 453, 596 (1995).

[74] M. T. Murphy, J. K. Webb, V. V. Flambaum, M. J. Drinkwater, F. Combes, and T. Wiklind, Mon. Not. R. Astron. Soc. 327, 1244 (2001).

[75] A. M. M. Pinho and C. J. A. P. Martins, Phys. Lett. B 756, 121 (2016).

[76] J. B. Whitmore and M. T. Murphy, Mon. Not. R. Astron. Soc. 447, 446 (2015).

[77] M. T. Murphy, A. L. Malec, and J. X. Prochaska, Mon. Not. R. Astron. Soc. 461, 2461 (2016).

[78] M. T. Murphy, A. L. Malec, and J. X. Prochaska, Mon. Not. R. Astron. Soc. 464, 2609 (2017).

[79] S. A. Levshakov, P. Molaro, S. Lopez, S. D'Odorico, M. Centurión, P. Bonifacio, I. I. Agafonova, and D. Reimers, Astron. Astrophys. 466, 1077 (2007).

[80] J. Darling, Astrophys. J. 612, 58 (2004).

[81] M. T. Murphy, J. K. Webb, V. V. Flambaum, M. J. Drinkwater, F. Combes, and T. Wiklind, Mon. Not. R. Astron. Soc. 327, 1244 (2001).

[82] N. Kanekar, C. L. Carilli, G. I. Langston, G. Rocha, F. Combes, R. Subrahmanyan, J. T. Stocke, K. M. Menten, F. H. Briggs, and T. Wiklind, Phys. Rev. Lett. 95, 261301 (2005).

[83] A. Songaila and L. Cowie, Astrophys. J. 793, 103 (2014).

[84] P. Molaro, D. Reimers, I. I. Agafonova, and S. A. Levshakov, Eur. Phys. J. Spec. Top. 163, 173 (2008).

[85] H. Chand, R. Srianand, P. Petitjean, B. Aracil, R. Quast, and D. Reimers, Astron. Astrophys. 451, 45 (2006).

[86] P. Molaro, M. Centurión, J. B. Whitmore et al., Astron. Astrophys. 555, A68 (2013).

[87] T. M. Evans, M. T. Murphy, J. B. Whitmore, T. Misawa, M. Centurion, S. D’Odorico, S. Lopez, C. J. A. P. Martins, P. Molaro, P. Petitjean, H. Rahmani, R. Srianand, and M. Wendt, Mon. Not. R. Astron. Soc. 445, 128 (2014).

[88] T. Damour and F. Dyson, Nuc. Phys. B480, 37 (1996).

[89] P. Sisterna and H. Vucetich, Phys. Rev. D 44, 3096 (1991).

[90] S. K. Lamoreaux and J. R. Torgerson, Phys. Rev. D 69, 121701 (2004).

[91] P. Sisterna and H. Vucetich, Phys. Rev. D 41, 1034 (1990).

[92] J. D. Prestage, R. L. Tjoelker, and L. Maleki, Phys. Rev. Lett. 74, 3511 (1995).

[93] Y. Sortais, S. Bize, M. Abgrall, S. Zhang, C. Nicolas, C. Mandache, P. Lemonde, P. Laurent, G. Santarelli, N. Dimarcq et al., Phys. Scr. T95, 50 (2001).

[94] H. Marion, F. Pereira Dos Santos, M. Abgrall, S. Zhang, Y. Sortais, S. Bize, I. Maksimovic, D. Calonico, J. Grünert, C. Mandache et al., Phys. Rev. Lett. 90, 150801 (2003).

[95] M. Fischer, N. Kolachevsky, M. Zimmermann, R. Holzwarth, Th. Udem, T. W. Hänsch, M. Abgrall, J. Grünert, I. Maksimovic, S. Bize et al., Phys. Rev. Lett. 92, 230802 (2004). 
[96] B. Li, D. F. Mota, and J. D. Barrow, Astrophys. J. 728, 108 (2011).

[97] L. Lorini, N. Ashby, A. Brusch, S. Diddams, R. Drullinger, E. Eason, T. Fortier, P. Hastings, T. Heavner, D. Hume, W. Itano et al., Eur. Phys. J. Spec. Top. 163, 19 (2008).

[98] P. Touboul, G. Métris, M. Rodrigues, Y. André, Q. Baghi, J. Bergé, D. Boulanger, S. Bremer, P. Carle, R. Chhun et al., Phys. Rev. Lett. 119, 231101 (2017).

[99] J.S. Levinger, Nuclear Photo-Disintegration (Oxford University Press, New York, 1960).
[100] B. L. Berman and S. C. Fultz, Rev. Mod. Phys. 47, 713 (1975).

[101] M. Gorchtein, T. Hobbs, J. Timothy Londergan, and A. P. Szczepaniak, Phys. Rev. C 84, 065202 (2011).

[102] L. Kraiselburd, M. Miller Bertolami, P. Sisterna, and H. Vucetich, Astron. Astrophys. 529, A125 (2011).

[103] H. Utsunomiya, S. Katayama, I. Gheorghe, S. Imai, H. Yamaguchi, D. Kahl, Y. Sakaguchi, T. Shima, K. Takahisa, and S. Miyamoto, Phys. Rev. C 92, 064323 (2015). 